\title{
Immediate Hypersensitivity Reaction Followed by Successful Oral Desensitization to Ursodiol
}

Erika Yue Lee ( $\nabla$ erika.lee@mail.utoronto.ca )

University of Toronto https://orcid.org/0000-0003-1108-2067

\section{Christine Song}

University of Toronto

\section{Case report}

Keywords: Immediate drug hypersensitivity, ursodiol, ursodeoxycholic acid, desensitization, primary biliary cholangitis

Posted Date: April 29th, 2021

DOl: https://doi.org/10.21203/rs.3.rs-459540/v1

License: (1) This work is licensed under a Creative Commons Attribution 4.0 International License. Read Full License

Version of Record: A version of this preprint was published at Allergy, Asthma \& Clinical Immunology on July 22nd, 2021. See the published version at https://doi.org/10.1186/s13223-021-00578-7. 


\section{Abstract}

Background: Immediate hypersensitivity reaction to ursodiol is rare and there is no previously published protocol on ursodiol desensitization.

Case presentation: A 59-year-old woman with primary biliary cholangitis (PBC) developed an immediate hypersensitivity reaction to ursodiol - the first-line treatment for PBC. When she switched to a second-line treatment, her PBC continued to progress. As such, she completed a novel 12-step desensitization protocol to oral ursodiol. She experienced recurrent pruritus after each dose following desensitization, which subsided after a month of being on daily ursodiol.

Conclusion: Immediate hypersensitivity reaction to ursodiol is uncommon. Our case demonstrated that this novel desensitization protocol to ursodiol could be safely implemented when alternative options are not available or have proven inferior in efficacy.

\section{Background}

Primary biliary cholangitis (PBC), previously known as primary biliary cirrhosis, is an autoimmune disease of the liver characterized by T-cell mediated attack of intralobular bile ducts [1, 2]. The eventual loss of these bile ducts results in cholestasis, which clinically manifests as fatigue, pruritus and jaundice [2]. Without treatment, PBC eventually leads to cirrhosis and liver failure.

Ursodiol or ursodeoxycholic acid (UDCA) is the mainstay treatment for PBC. It is currently one of the only two approved therapies for PBC; the other therapy is obeticholic acid, which is considered second-line [3]. Ursodiol delays the progression to end-stage liver disease, prolongs survival and is generally well tolerated (3). Patients who fail to respond to or do not tolerate the first-line ursodiol can have or switch to obeticholic acid [3]. Infrequent adverse effects of ursodiol include diarrhea, weight gain and paradoxically pruritus [4], but hypersensitivity to ursodiol has not been reported. Herein we describe a patient with PBC who developed an immediate allergic reaction to ursodiol.

\section{Case Presentation}

A 59-year-old woman who did not have any allergic or atopic history was diagnosed with PBC. She was started on ursodiol $500 \mathrm{mg}$ twice daily at the time of her diagnosis. On Day 4 of therapy, she developed full-body hives, chest tightness and dyspnea within one hour of her dose. These symptoms self-resolved within an hour. Subsequently she lowered the dose of ursodiol to $250 \mathrm{mg}$ twice daily at the recommendation of her hepatologist. On Day 2 of re-exposure, she developed similar but more severe symptoms within an hour of her dose. These symptoms resolved without treatment within an hour. Consequently, ursodiol was discontinued by her hepatologist and the patient was referred to the allergy clinic for ursodiol allergy. 
At our clinic, we confirmed the history of her systemic reactions to ursodiol. Allergy testing was not done, as there is no validated skin testing or available serum IgE measurement for ursodiol. Patient declined drug provocation test due to fear of reaction. As such, the diagnosis of an IgE-mediated reaction to ursodiol was made based on detailed clinical history. Given the ongoing need for ursodiol, we recommended the patient to undergo desensitization. She declined the procedure due to personal reasons, so was switched to the second-line obeticholic acid for her PBC. In the following year, her PBC continued to progress and she was receptive to desensitization. Her hepatologist re-referred her to our clinic for consideration of desensitization to ursodiol.

A new ursodiol desensitization protocol was developed (Table 1). The solution was made by our inpatient hospital pharmacy. Patient successfully completed the protocol in the intensive care unit and has remained on ursodiol $500 \mathrm{mg}$ twice daily. Following desensitization, patient initially experienced recurrent pruritus each time after taking ursodiol. A careful evaluation in the clinic did not raise any concerns for an allergic reaction. After a discussion with the patient, she agreed to continue taking ursodiol and pruritus was somewhat reduced with non-sedating antihistamine. A month after the desensitization and being on daily ursodiol, patient reported a complete resolution of her pruritus and was able to tolerate ursodiol without any issues.

Table 1

Ursodiol desensitization using a 12-step, 2-dilution protocol.

\begin{tabular}{|lllllll|}
\hline Step & $\begin{array}{l}\text { Concentration } \\
(\mathrm{mg} / \mathrm{mL})\end{array}$ & $\begin{array}{l}\text { Time } \\
(\mathrm{hr}: \mathrm{min})\end{array}$ & $\begin{array}{l}\text { Route of } \\
\text { administration }\end{array}$ & $\begin{array}{l}\text { Volume given } \\
\text { per step }(\mathrm{mL})\end{array}$ & $\begin{array}{l}\text { Dose given } \\
\text { per step }(\mathrm{mg})\end{array}$ & $\begin{array}{l}\text { Cumulative } \\
\text { dose }(\mathrm{mg})\end{array}$ \\
\hline 1 & 5 & $00: 00$ & Oral & 0.05 & 0.25 & 0.25 \\
\hline 2 & 5 & $00: 45$ & Oral & 0.1 & 0.5 & 0.75 \\
\hline 3 & 5 & $01: 30$ & Oral & 0.2 & 1 & 1.75 \\
\hline 4 & 5 & $02: 15$ & Oral & 0.4 & 2 & 2.75 \\
\hline 5 & 5 & $03: 00$ & Oral & 0.8 & 4 & 6.75 \\
\hline 6 & 5 & $03: 45$ & Oral & 1.25 & 6.25 & 13 \\
\hline 7 & 5 & $04: 30$ & Oral & 2.5 & 12.5 & 25.5 \\
\hline 8 & 20 & $05: 15$ & Oral & 1.25 & 25 & 30.5 \\
\hline 9 & 20 & $06: 00$ & Oral & 2.5 & 50 & 80.5 \\
\hline 10 & 20 & $06: 45$ & Oral & 3.75 & 75 & 155.5 \\
\hline 11 & 20 & $07: 30$ & Oral & 7.5 & 150 & 305.5 \\
\hline 12 & 20 & $08: 15$ & Oral & 10 & 200 & 505.5 \\
\hline Target dose of $500 \mathrm{mg}$. & & & & & \\
\hline
\end{tabular}




\section{Discussion And Conclusions}

Ursodiol is a hydrophilic bile acid that can increase the metabolic conversion of cholesterol to bile acids, thus reducing the fractional reabsorption of cholesterol by intestines [5]. Its mechanisms of action in PBC are not clearly defined, but the proposed mechanisms include increasing the hydrophilicity in the circulating bile acid pool, stimulating hepatocellular and ductal secretions, protecting against bile acidand cytokine-induced injury, and exerting immunodulating and anti-inflammatory effects $[5,6]$. Although ursodiol has been the standard of care for patients with PBC for years, up to $40 \%$ of patients fail to respond to it with inadequate biochemical improvement and thus are still at risk of progression to endstage liver disease [7]. Although we do not know how our patient would have responded to ursodiol following desensitization from the liver standpoint, the worsening of her PBC while being on obeticholic acid prior to desensitization warranted her to switch to ursodiol.

Ursodiol is generally well tolerated and shown to improve pruritus in patients with PBC [6]. Paradoxically, exacerbation of pruritus after ursodiol administration has also been reported [4]. Our patient who developed pruritus after desensitization likely experienced a medication side effect as opposed to an allergic reaction in the absence of objective findings. Further, although delayed hypersensitivity reaction to ursodiol, manifesting as skin rash with biopsy-proven lichenoid reaction, has been reported [8], an immediate hypersensitivity has not been reported in the literature.

To our knowledge, this is the first reported case of an immediate allergic reaction followed by successful oral desensitization to ursodiol. We propose that this 12-step desensitization protocol to ursodiol can be safely implemented when alternative options are not available or have proven inferior in efficacy.

\section{Abbreviations}

PBC, primary biliary cholangitis

UDCA, ursodeoxycholic acid

\section{Declarations}

Conflict of interest: none

Ethics approval and consent to participate: not applicable

Consent for publication: Patient consent was obtained.

Availability of data and materials: not applicable

Competing interests: none 
Funding: none

Authors' contributions: EL involved in drafting and submitting the manuscript. CS involved in drafting the manuscript.

Acknowledgements: not applicable

\section{References}

1. Kaplan MM. Primary biliary cirrhosis. N Engl J Med. 1996;335:1570-80.

2. Lindor K. Ursodeoxycholic acid for the treatment of primary biliary cirrhosis. $\mathrm{N}$ Engl J Med. 2007;357:1524-9.

3. Corpechot C, Abenavoli L, Rabahi N, Chretien Y, Andreani T, Johanet C, et al. Biochemical response to ursodeoxycholic acid and long-term prognosis in primary biliary cirrhosis. Hepatology. 2008;48:8717.

4. Hempfling W, Dilger K, Beuers U. Systematic review: ursodeoxycholic acid-adverse effects and drug interactions. Aliment Pharmacol Ther. 2003;18:963-72.

5. Gulamhusein AF, Hirschfield GM. Primary biliary cholangitis: pathogenesis and therapeutic opportunities. Nat Rev Gastroenterol Hepatol. 2020;17:93-110.

6. Poupon R. Ursodeoxycholic acid and bile-acid mimetics as therapeutic agents for cholestatic liver diseases: an overview of their mechanisms of action. Clin Res Hepatol Gastroenterol. 2012;36(Suppl 1):3-12.

7. Lammers WJ, van Buuren HR, Hirschfield GM, Janssen HL, Ivernizzi P, Mason AL, et al. Levels of alkaline phosphatase and bilirubin are surrogate end points of outcomes of patients with primary biliary cirrhosis: an international follow-up study. Gastroenterology. 2014;147:1338-49.

8. Horiuchi Y. Lichenoid eruptions due to ursodeoxycholic acid administration. Gastroenterology. 2001;121:501-2. 